\title{
De l'oralité au récit: le cas du livre sacré des indigènes Sateré-Mawé, en Amazonie
}

\author{
Renan Albuquerque \\ Universidade Federal do Amazonas, Faculdade de Informação e Comunicação, Manaus, AM, Brasil \\ ORCID: https://orcid.org/0000-0002-3923-9938
}

\section{Fabrício Vasconcelos}

Universidade Federal do Amazonas, Manaus, AM, Brasil

ORCID: https://orcid.org/0000-0001-9919-5642

\section{Flávia Roberta Busarello}

Pontifícia Universidade Católica de São Paulo, Programa de Psicologia Social, São Paulo, SP, Brasil ORCID: https://orcid.org/0000-0002-3384-9305

\begin{abstract}
Résumé
L'objectif a été de (i) caractériser le processus narratif et communicationnel, né de l'oralité, d'une œuvre sacrée, de référence des Sateré-Mawé, et (ii) effectuer des lectures et des analyses interprétatives des textes du livre, avec analyse de contenu, en considérant la communication orale et le discours. Ces deux points sont décrits dans les rubriques "Approche Théorique » et dans la partie des " Résultats et Discussion ». La présentation de la mythologie des Sateré-Mawé est claire dans sa conception historique, car dans le Sehaypóri..., qui signifie " recueil de mythes ", ce qu'on a souligné a été surtout des symboles et la façon dont ce peuple construit leurs mémoires autrefois transmises oralement.
\end{abstract}

\section{Mots-clés}

Oralité; Récit; Littérature; Sateré-Mawé/AM; Amazonie

\section{Introduction: I’andirá-marau et l'ethnomidialogie impliquée}

Élément important pour comprendre les sociétés des terres basses d'Amérique du Sud et leurs expériences historiques c'est la littérature. L'activité littéraire peut aider à l'interprétation de la condition humaine des amérindiens. Dans ce sens, nous soulignons l'oeuvre Sehaypóri: le livre sacré du peuple Sateré-Mawé. Dans ce livre, l'auteur Yaguarê 
Yamã présente des récits liés aux savoirs ethniques du peuple Sateré-Mawé de la Terre Indigène (TI) Andirá-Marau, en Amazonie centrale.

Yamã est indigène et, à partir de cette oeuvre, il présente des éléments importants pour que nous puissions identifier les connaissances de son ethnie. C'est une littérature née de l'oralité, stratégique pour la communication des Sateré-Mawé avec les blancs. Elle facilite le partage d'informations relatives aux nations amazoniennes en général.

Pour aborder théoriquement la perspective, nous soulignons l'ethnomidialogie (FERREIRA, 2012). Le courant théorique s'est développé au Brésil et étudie les instruments informatifs et médiatiques utilisés par les amérindiens pour donner de la publicité à leur réalité. Ainsi, par le biais de l'ethnomidialogie, nous avons étudié dans quelle mesure Yamã, dans Sehaypóri..., a utilisé des éléments de sa propre culture pour raconter son histoire.

$\mathrm{Au}$ moyen d'un support théorique, l'objectif a été de vérifier l'importance de la communication ethnomidialogique dans le processus de construction de la personne et de la mémoire indigène. Nous sommes partis de l'hypothèse que, en ce qui concerne la communication écrite et orale, il est possible d'observer l'existence d'un discours littéraire ancré dans les faits, les résultats d'expériences et les assimilations. De plus, dans Sehaypóri... nous avons l'indigène d'avant la conquête décrit à partir de son propre regard.

A partir de l'hypothèse, nous avons effectué i) la caractérisation du processus narratif et communicationnel né de l'oralité du texte et de la ligne de développement de l'oeuvre clé rapportée et ii) des lectures interprétatives et des analyses des textes en considérant l'oralité, la communication et le discours (VALA, 1986; BARDIN, 2002). Ces points sont décrits dans les « Approche Théorique » et « Résultats et Discussion ».

\section{Approche théorique: l'ethnomidialogie}

L'ethnomidialogie est une théorie authentiquement brésilienne du domaine de la communication sociale (FERREIRA, 2012). Elle est axée sur l'analyse des processus de communication des peuples autochtones, y compris les amérindiens d'Amazonie. A partir de l'ethnomidialogie, il a été possible de réaliser des études scientifiques et médiatiques des matrices sociales précolombiennes (IANNI, 1999).

L'ethnomidialogie a pour objectif de servir de base à l'étude de la communication, écrite ou orale, à travers une approche interdisciplinaire. L'ethnomidialogie s'attache à 
comprendre la transmission d'informations provenant de sociétés culturellement diverses et les impacts de cette diffusion, comme l'ont initialement suggéré Sperber et Wilson (1995).

L'orientation du concept d'ethnomidialogie s'est renforcée dans la mesure où l'on a supposé que la communication sociale contribuait au renforcement des contextes historiques et culturels des peuples traditionnels et originaires. Ainsi, l'ethnomidialogie est devenue une branche de l'étude de la communication qui tend à étudier les bases de la diversité ethnique nationale et à développer des pratiques communicationnelles interactives, en semant des liens entre les grands groupes médiatiques et les peuples ethniques (MENDONÇA et al., 2007).

Dans notre cas particulier, lorsque nous nous basons sur ce modèle d'analyse de la communication, l'objectif était de comprendre les phénomènes sociaux et culturels des Sateré-Mawé. Pour cette raison, dans la corrélation entre la contribution théorique et l'objet d'étude, nous avons traité les pratiques communicationnelles des peuples indigènes de la Terre Indigène Andirá-Marau liées au développement littéraire développé dans l'intérieur (FERREIRA, 2015).

Le choix référentiel de Sehaypóri... nous a permis de voir dans quelle mesure la diffusion d'informations (VOSOUGHI; ROY; ARAL, 2018) tend à contribuer à une plus grande connaissance de la société en général sur la socioculture de l'ethnie. Dans l'oeuvre, Yamã montre sa reconnaissance personnelle des amazonités (mode de vie en Amazonie) composantes du contexte Sateré-Mawé et communique à travers des univers extrahumains, en mettant en valeur ses connaissances millénaires et ancestrales.

Évaluer le processus de communication à partir du livre a été la première étape pour penser les mécanismes de transmission et de maintien de l'histoire des Sateré-Mawé à travers l'ethnomidialogie. Par conséquent, à notre avis, l'ethnomidialogie a été un moyen théorique important pour élucider des complexités de la recherche en communication sociale qui se concentre sur les peuples autochtones (WAGNER, 2010).

\section{Méthodologie}

L'étude a été qualitative, via approche transversal, appliquée à des pratiques communicationnelles et des implications de ces pratiques à partir de Sehaypóri..., d'après Yaguarê Yamã. Le texte décrit les modes de vie et les engendrements de la réalité Sateré- 
Mawé, en mettant l'accent sur les indigènes de la TI Andirá-Marau, située dans la divise de l'État de l'Amazonas avec l'État du Pará.

L'étude de Sehaypóri... a été effectuée à partir de la lecture de l'œuvre par analyse de contenu. Au premier moment, nous avons eu le contact avec des textes construits par Yaguarê, raconté à lui, tout au long du livre, en cherchant comprendre la façon dont il s'est modelé et a également modelé les Sateré-Mawé tandis que construction de la pensée liée à la personne indigène. Dans la deuxième étape, nous avons inféré comme Yaguarê a présenté la relation de l'oralité et du savoir entre deux sociétés distinctes: celle des Sateré-Mawé et celle des blancs. Dans la dernière étape, nous avons effectué des stratégies de communication pour la compréhension concernant les mythes décrits.

Les récits du livre ont été évalués en fonction d'une tendance sémantique et représentation significative (ABRIC, 2005). Grâce à la visibilité des occurrences ou cooccurrences communicationnelles, les dimensions de l'oralité abordées par Yamã ont été interprétées. Nous avons utilisé des lectures de biais flottant et inférentielle pour réaliser l'activité.

\section{Résultats et discussion}

Dans le livre, on décrit un ensemble de mythes qui partent de l'ascendance socioculturelle des Sateré-Mawé. Dans Sehaypóri... les spiritualités de l'ethnie sont explorées en fonction de leurs croyances cosmologiques. Le thème de l'oeuvre tourne autour de la vie et de la mort des indigènes. Et nous comprenons que l'idéede « vie et mort » est incluse dans la proposition parce que dans le livre Yamã était intéressé à étudier le plan métaphysique de la société de l'Andirá-Marau.

L'écrivain construit ses points de vue en indiquant des conceptions et des perspectives du monde selon les notions de réalité que possèdent les Sateré-Mawé. De même, il décrit dans les récits du livre la constitution de l'imaginaire de l'ethnie. Par conséquent, les histoires de Sehaypóri... sont plus que des enregistrements d'une oralité amérindienne. Elles représentent une forme avancée de transmission de connaissances. Cela signifie que le caractère ethnomidialogique de la littérature amérindienne, à travers l'oeuvre de Yaguarê, semble se matérialiser à tel point que la traduction de la réalité se fait à partir de l'écriture et de la communicabilité de son oeuvre. 
Le livre ne vise pas seulement à faire une épistémologie explicative des causes et des choses que l'ethnie comprend, mais il confronte la logique blanche et occidentale sur les représentations humaines actuelles. Yamã parle beaucoup de lui-même en mettant en avant les territorialités et les symbologies ancestrales. L'expression de ces mythes impressionne à travers la teneur emblématique des mots, phrases, titres, de chaque chapitre du livre. La brochure est divisée en trois chapitres.

Tout d'abord, dans Ehay Tupã, les mythes sur les « créature du fond » sont discutés. Ces « créatures du fond » sont en fait des êtres humains qui se sont transformés en animaux aquatiques et ont commencé à avoir des pouvoirs surnaturels. Aujourd'hui, ils peuvent s'adresser aux populations autochtones qui n'ont pas subi la même transmutation et ne vivent pas sous l'eau.

Une autre question abordée par Yamã dans le premier chapitre fait référence aux lois et aux commandements du biome impliquant les indigènes et le soin de la Terre Mère, dans le cadre d'une schématisation dualiste du bien et du mal, où un Dieu du Bien est représenté par Tupãna et un Diable du Mal par Yurupary. Ici, il faut le noter, on remarque des similitudes entre l'oeuvre indigène et la littérature chrétienne - ce qui n'est pas nécessairement positif ou négatif, c'est juste un fait. Comme dans les récits de la Bible chrétienne, l'oralité du livre Sehaypóri... présente une écriture perspectiviste (VIVEIROS DE CASTRO, 1998, 2007).

Les personnages insérés sont des animaux familiers, tels que des poissons, des oiseaux et des bêtes aquatiques, qui apparaissent comme des personnes dotées d'une conscience mais sous forme animale. Ils sont capables de juger les autres et ont des histoires similaires à la vie sociale des humains. Les "créatures du fond " peuvent également être qualifiées " d'enchantées ». Ces êtres non humains mais conscients d'eux-mêmes sont connus non seulement dans la culture Sateré-Mawé, mais dans une grande partie du savoir régional amazonien. On trouve même une référence aux « créatures du fond » dans les Dauphins Roses d'Amazonie, qui portent avec eux une charge mythique expressive, qui leur confère une représentativité ethnomidialogique.

Il y a des passages que nous renvoient au fait sur l'usage de la littérature, dans les cadres ethnomidialogiques, tend d'aider ceux qui ne possèdent pas de proximité avec les histoires Sateré-Mawé à pénétrer dans l'univers immémorial des indigènes. À un seul temps, la narrative d'Yamã contribue pour démocratiser le savoir amérindien et fait également que 
nous soyons forcés à revoir des concepts de l'ici et maintenant. L'ethnomidialogie engendre des interprétations que les non-indigènes peuvent faire, telles que la démocratisation des savoirs amérindiens, la popularisation de ses modes de vie, la diffusion de mythiques primordiales de l'ethnie référenciée et la dissémination de conscience de l'autre.

Par exemple, selon les contes de l'œuvre, l'origine du cèdre du Brésil (timbó), de la guaraná et de la châtaigne, outre la propre humanité, a son apogée dans les conflits entre des êtres détenteurs de pouvoirs métaphysiques et humains débutés et non-débutés (des chamans et des indigènes qui ne possèdent pas de constitution et formation en magie ethnique).

La Bible raconte l'exode du peuple choisi par Dieu vers une terre promise, nommée Canaan. Guidés par l'orientation divine, ils ont marché par le désert pendant très longtemps, entourés d'entraves et difficultés, mais ils ont vaincu les adversités et, selon la narration dans le livre sacré des chrétiens, ils ont prospéré. Sehaypóri... relate l’exode des Mawé, peuple choisi par Tupãna, le dieu du bien et des vérités universelles, dans son parcours par l'Amazonie jusqu'à la nouvelle Mawézia - la patrie des Mawé. Yurupary, dieu du mal, malgré exercer forte influence dans la tentative de frustrer l'arrivée des Mawé à la terre promise, n'a pas obtenu succès. Car, hordes de tribus, non appliquées pour les êtres mythologiques et hostiles, tels que les Jumas (géants qui écrasaient les ennemis avec les pieds) et les Mapinguarys (monstres gardiens du portail Aryá-Wató) ont étés quelques-uns des empêchements pendant l'exode des Mawé.

Après l'arrivée des peuples Mawé à la terre promise, comme nous raconte les écrits, ils vivaient en harmonie dans la forêt amazonienne, jusqu'à un jour qu'un Yurupary a décidé d'anéantir tous les animaux de la surface du globe, mettant en danger la vie du peuple Mawé. Dans un épisode inusité durant une fête de mariage, Heté-Wakóp, un fiancé trahi par son épouse, décide de se venger de la trahison. Les esprits de la nature ont aidé le jeune homme en élevant un guerrier mythique afin de transformer tous les invités en animaux. Et ainsi il y a eu la création des différentes bêtes qui autrefois ont été détruites par Yurupary. C'est-à-dire, ici nous avons traité d'une naissance de l'humanité en raison de l'animalité terrestre, dans une claire note à la relation homme-animal.

Par le biais de l'ethnomidialogie, nous interprétons le livre et en extrayons également la signification suivante: la relation entre l'ethnie et le monde des blancs est guidée par des symboles. La complexité de cette affirmation suggère l'existence d'un 
ensemble d'émotions qui intègrent la vie quotidienne de cette société autochtone. L'amour, la haine, le bonheur, l'angoisse et l'indifférence. C'est exactement ce que nous avons remarqué en tentant de démontrer la quantité de contenu que nous pouvons extraire et évaluer grâce à l'ethnomidialogie, la démocratisation de la connaissance des peuples originaires.

Un autre cas fréquent dans l'ouvrage fait référence au Puratig Sa'Amy. Le Puratig est un objet sacré, un totem. Il a les caractéristiques d'un aviron et, pour les Sateré-Mawé, selon la tradition, il protège de l'oubli les mythes et les jalons temporels de l'ethnie. Dans le livre, Enumaré'hi'yt est un tuxaua légendaire qui protégeait l'aviron sacré des mains des démons afin qu'ils ne fassent pas mauvais usage de ce puissant instrument. On pense qu'aujourd'hui encore, il existe deux exemplaires du Puratig sur le territoire Sateré-Mawé, qui est le plus grand symbole de leur culture. Son usage traverse des lieux légendaires et des mondes submergés, gardant la connaissance de l'univers de l'ethnie et des lois de la nation, selon son histoire millénaire.

Les Sateré-Mawé, qui préservent la culture du guaraná, sont également connus pour le Rituel de la Tucandeira. Ils pratiquent cette activité depuis des millénaires et, selon Yaguarê, l'origine de ce rituel est très ancienne. Tout a commencé avec les démons qui utilisaient des animaux venimeux comme les araignées, les scorpions, les serpents et les millepattes pour piquer et produire de la force par la douleur. À partir de cette idée, les gens ont suivi la tradition du conflit rituel pour les avantages offerts par l'acte. On croit que les injections apportent la guérison au peuple: les garçons deviennent des hommes guerriers prêts à pêcher, chasser et combattre après le rituel des injections.

Lors du rituel de la Tucandeira, plusieurs fourmis sont placées dans un gant fait de paille de tucum. Elles sont connues sous le nom de fourmis du Cap-Vert et ont une piqûre douloureuse, qui apporte un malaise à l'individu choisi pour l'initiation.

L'origine du rituel a eu lieu pendant la recherche par le frère cadet du grand Tatu, qui avait s'est perdu dans la forêt. Celui-ci avait sorti pour chercher un animal qui puisse lui piquer et, ainsi, il deviendrait un grand chasseur, comme son frère ainé, cependant il s'est retrouvé tout seul dans le bois. La découverte des origines du rituel a eu lieu dans une caverne où habitait la Grande Jiboia - lorsque le grand-frère cherchait le frère cadet -, appelée également de Mère-des-Serpents, qui avait la fourmi Tucandeira comme poux dans les poils des organes génitaux. Après avoir pris les fourmis Tucandeiras et les placer toutes 
dans un gant tressé en fibres, le frère ainé du grand Tatu est rentré à la maison et livre à son frère cadet, qui avait déjà y rentré. Après l'épisode, des plumes d'ara rouge ont été ajoutées avec des plumes de faucon et ensuite la danse a été créée pour accompagner le rituel de piqures.

Remarquons que l'option pour décrire l'origine du mythe est usuelle, retirée du quotidien indigène, à partir d'un fait commun (se perdre dans le bois), mais il existe un enlace de composition de la narrative qui rend accessible la compréhension de la mythique à propos du rituel. La stratégie est une action ethnomidialogique de dissémination de ce savoir hybride, surtout dans le sens que la théorisation s'ancre du traitement avec la réalité racontée, qu'elle soit symbolique ou territorialement décrite. Et raconter des histoires, pour la sagesse amérindienne, c'est plus que transmettre des informations déconnexées, est enseigner en revisitant des sagesses profondes.

À la fin du chapitre sur les Mythes Sacrés du livre, il devient clair que les entités nommées par les Sateré-Mawé comme Ipakahawag Sehay sont des êtres qui vivent dans un monde submergé. Elles peuvent être des dauphins roses d'Amazonie, par exemple, qui se transforment en humains. La légende du dauphin (rose ou tucuxi) raconte l'histoire d'un homme qui part à la recherche de nourriture pour sa famille et qui, en pêchant, aperçoit un dauphin d'Amazonie qui s'est éloigné d'un banc. Il lui lance un harpon dans le dos, sans imaginer qu'il s'agit d'une femelle, la «femme » d'un dauphin. Cherchant à se venger, le « mari » du dauphin femelle (sa femme assassinée) a décidé de se rendre chez l'homme pour l'amener au fond de la rivière.

Après un procès tenu dans le monde enchanté des marsouins, le meurtrier a été condamné par le marsouin. Mais la femme de l'humain n'a pas permis cela et l'a supplié de le libérer de ce monde souterrain. La décision du tribunal a été de libérer le mari meurtrier, mais à la condition qu'il ne tue plus d'animaux et se nourrisse uniquement de fruits et de racines. Le couple devrait également rester éloigné de tout endroit rempli de sang. Aujourd'hui encore, on parle d'une cité sous-marine enchantée, mais on pense que seuls les chamans peuvent visiter le monde sous-marin sans courir le risque de devenir un marsouin.

Dans l'extrait en analyse, encore une fois la figure du langage métaphorique, soutenue dans historicité de l'ethnie, est utilisée pour la transmission de connaissances. La perspective du devenir, parmi humains et non-humains, homme et boto, est présentée pour l'interprétation d'une dispute entre les êtres des eaux et les êtres de la forêt. 
La théorie ethnomidialogique pour vérifier et pondérer sur la sagesse relatée concernant les gens et les animaux nous aide à réaliser à quel point l'inventivité de l'auteur s'utilise pour projeter l'histoire de l'ethnie en mode de littérature. Également dans le chapitre 2, intitulé Sehay Sése - Les légendes, est abordée dès l'origine du cajou jusqu'à la vengeance du fils de Gavião-Homme. Ce mythe qui a caractère fictive, mais haute relevance subjective, étant transmis grâce à un contexte de réalité autrefois vécue. Il convient de noter que la narrative a du composant fantastique interposé. Et cela, naturellement, est un biais de l'ethnomidialogie: transmettre des savoirs à partir de l'histoire, mais avec un biais de fictionnisme ayant en vue de devenir plus intéressant ce qui est narré.

Dans la première légende narrée au deuxième chapitre l'on pointe l'apparition d'une plante populaire de l'Amazonie, grâce à ses fruits, le cajou. L'arbre responsable pour la génération a eu son apparition jadis, dans le territoire des Sateré-Mawé. L'histoire raconte sur un jeune guerrier, appelé piahãg-saike, dont la naissance a été prédite par les painis (chamans) du village, guidés par la divinité de Tupãna. La naissance du nouveau guerrier appartenant au clan ut (sateré) a eu lieu aux rives du Fleuve Andirá, actuellement connu comme "Freguesia do Andirá », localisé dans la commune de Barreirinha, distante 372 kilomètres de Manaus/AM. Piahãg-saikê a été l'un des dirigeants choisis du village pour protéger son peuple.

Il est possible de percevoir l'exaltation d'hommes appartenant à la tribu SateréMawé, considérés des braves guerriers. Mais après une guerre contre les Parintintim, qui essayaient de dominer le territoire, le guerrier piahãg-saike, qui avait été déclaré le futur tuxaua-général, meurt avec une lance ennemie traversée dans sa poitrine. Malgré tout cela, ils remportent la guerre contre les Parintintim. Or, la morte inattendue a créé une commotion dans le village. Les funérailles de piahãg ont été dans un des rives du sacré Fleuve Andirá et, après longtemps passé, il est née une plante rare au-dessus de la tombe du guerrier, le cajoueiro. Tous les villageois ont compris que s'agissait du jeune piahãg-saike qui avait né de nouveau sous forme d'arbre, en devenant l'arbre sacrée.

En prenant les dispositions en tant que stratégies de communication à partir d'éléments historiques, l'auteur traite la réalité et la fiction de façon continue, en rassemblant dans une seule narrative des aspects réels et fantastiques de l'univers SateréMawé, ce qu'enrichit la perspective de lecture puisque propose une symbiose entre ce que s'est passé en fait et ce que l'auteur voulait qu'avait s'est passé en projection. Il s'agit d'une 
non dissociation primordiale. La prédisposition rend la chaine d'évènements complexe dans laquelle des faits ou fictions sont décrites.

Il est important de souligner que l'ethnomidialogie offre de nombreuses possibilités de diffusion socioculturelle. Yaguarê Yamã, par exemple, utilise cet apport théorique pour composer sa littérature indigène. Le savoir indigène qu'il diffuse à partir de ce cadre théorique est composé d'une collectivité organique qui intéresse les peuples indigènes. Il ne s'agit pas seulement de la position individuelle de l'écrivain, mais du point de vue de toute une ethnie, avec sa base cosmologique, clanique et de parenté. Il ne nous semble pas exagéré d'affirmer que Yaguarê utilise une base conceptuelle ethnomidialogique pour retracer toute sa trajectoire d'écrivain indigène d'ascendance Sateré-Mawé associé aux Maraguá.

Dans un autre cas, celui de l'origine du manioc, il y a une histoire curieuse dans laquelle le Tuxaua des Jaguars, Awayató-Pótavait une fille nommée Ywerói, elle attirait l'attention par sa beauté, et, comme un père jaloux, Awayató-Pótétait craintif de cette situation. Un jour, le crapaud Ó'ok a dit à sa grand-mère qu'il était destiné à conquérir le cœur de Ywerói, mais elle ne voulait pas que son petit-fils risquait sa vie, car elle savait comme c'était perfide Awayató-Pót, il tuait tous ceux qui voulaient la marier. L'insistance d'Ó'ok était incontestable et, décidé à se marier avec la fille du Tuxaua des Jaguars, il est allé chez les Ywerói, dans les proximités du Fleuve Marau.

Le crapaud Ó’ok a appelé les habitants de la maison et soudain Awayató s'est tenu aux aguets, en attendant le bon moment pour attaquer l'indésirable visitant. Le Tuxaua des Jaguars a demandé à l'ó'ok d'entrer, mais en savant des mauvaises intentions du futur beaupère il a décidé de rester dehors.

Dans un moment donné le crapaud s'est souvenu de son ami le vent et lui a demandé d'être mis dedans la maison sans passer par la porte d'entrée. Entrant dans la chambre de sa fille, le Tuxaua des Jaguars fut surpris par la présence indésirable du crapaud. Sans savoir comme agir, Awayató-Pót a décidé d'attendre le lever du jour pour remettre son plan d'attaque contre le crapaud ó'ok. Le lendemain, de la même façon dont il est entré, le crapaud est sorti de la maison à l'aide du vent. Furieux, Awayató-Pót a décidé de poursuivre le crapaud et il a finir par être tué et son corps traíné pour le rive d'un ruisseau, étant transformé en crocodile en raison de son cadavre être sous les feuilles magiques du taperebá. 
Ywerói, fille du décédé Tuxaua des Jaguars, était enceinte de son mari Mãgká'i, frère du crapaud Ó’ok, et donc a décidé d'aller à la rencontre de son père, qui s'est transformé en crocodile, elle a été conseillée de ne pas se rapprocher de la maison de leurs oncles, en raison d'être, eux, intentionnés de se venger par la mort d'Awayató-Pót. Sans suivre les conseils de son mari, Ywerói est allée chez ses oncles et, en arrivant, est tombée morte après un sortilège lancé par leurs oncles. Puis ils l'ont baignée avec des feuilles magiques, et le corps de la nièce s'est transformée en manioc.

Il a été retiré du sang d'Ywerói le tukupy, qui s'est converti en jaune et, du fils qu'elle apportait dans son ventre, est apparu le tapioca à travers la viande. Aujourd'hui, il existe plusieurs dérivés du manioc, qui sont produits dans différentes régions et sont présents dans sa majorité dans les plats typiques de la région, tels que la farine d'eau, la farine sèche, le beiju-peteka et etc.

\subsection{Comparaison Entre Mythes}

Le contexte qui nous est présenté indique qu'un aliment traditionnel, également utilisé comme aliment rituel, est issu d'un conflit intrafamilial et interhumain. Il s'agissait d'une situation formée d'une triade d'éléments dynamiques, avec lesquels ils interagissaient et, par conséquent, on a observé qu'il y avait l'inclusion d'éléments magiques dans le processus. Cela nous montre que le fantastique, largement connu dans le domaine des lettres universelles en tant que genre littéraire, est à nouveau présent dans le récit de ce mythe.

Il est important de souligner que ce mythe en question est différent d'un autre, aussi ou même plus important pour les Sateré-Mawé. Nous parlons du mythe du jaguar et de la cutia (petit rongeur) (ALBUQUERQUE; JUNQUEIRA, 2017), qui ne travaille pas avec des questions fantastiques, mais plutôt avec la parenté et la base totémique. Pour cette raison, il nous est possible de faire la comparaison suivante: il y a les mythes d'un large ordre surnaturel et métaphysique, qui diffusent des valeurs à travers le biais imaginaire de l'ethnie ; et les mythes qui décrivent des passages historiques, à travers des constructions mémorielles.

Cette différence est marquée dans la légende des trois beaux-frères de Mãgká'i, qui raconte l'histoire d'un jeune crapaud qui vivait seul. Mãgká'i avait trois sœurs, mais il ne les connaissait pas, et un jour quelqu'un lui a donné mystérieusement un chapeau et une paire 
de sandales, qui avaient des pouvoirs magiques de téléportation. En mettant les sandales et le chapeau sur sa tête, Mãgká'i a fermé les yeux et en les ouvrant il était avec sa sœur ainée. Saluant sa sœur, il attend que son mari arrive pour le rencontrer. Le premier beau-frère du crapaud était le Faucon qui, à son arrivée, lui a offert de la nourriture et aussi un de ses ongles en cas d'urgence. Après avoir fait ses adieux, Mãgká'i s'est rendu chez sa deuxième sœur, qui était la femme du Jaguar Awayató, et selon le même procédé que son premier beau-frère, le Jaguar lui a offert un de ses ongles pour donner de la force au crapaud. Et enfin il est arrivé à sa dernière sœur, qui était mariée à Wawartó le Tapir, et pour faire plaisir à son beau-frère, Wawartó lui a aussi offert ses ongles pour sa protection. Après avoir fait un rêve étrange au sujet d'une jeune femme, Mãgká'i a utilisé son pouvoir pour retrouver cette femme qui semblait amère car elle avait été victime d'un sortilège.

Dans la description, on remarque clairement le facteur imaginaire beaucoup plus fort que la constitution historique ou mémorielle du récit. Et la raison humaine-animale, une fois de plus, est présentée comme le cœur de l'ethno communication. Sinon, voyons ce qui suit: la jeune fille était dans les profondeurs du fleuve Mamuru, d'autre part l'inconnue avait pour mari le serpent enchanté Mói'ók, une des filles de la vieille Mère des Serpents, Magkarú-Sése. Cependant, pour rendre la liberté à la jeune fille inconnue, il fallait accomplir une mission ardue, dont l'objectif était de trouver à la source de la rivière Mamuru le cœur du grand serpent caché dans une pierre. Les ongles que le crapaud Mãgká'i a reçues de ses beaux-frères lui ont permis d'invoquer chacun d'entre eux et de briser le cœur du Grand Serpent. Après la conquête, la liberté de la jeune fille a été réalisée et elle a épousé Mãgká'i, le crapaud.

En synthèse, nous avons la construction littéraire classique de «l'histoire aux conséquences édifiantes », largement utilisée par Yaguarê dans les récits qu'il met en valeur dans ses œuvres. Par conséquent, il est lucide de supposer que, comparativement, ce mythe aborde des perspectives fantastiques, surréalistes - différentes de l'historicité centrée sur le mythe du jaguar et de la cutia, comme nous l'avons souligné - préservant la triade i) héros, ii) antagoniste ou antihéros et iii) un acte de rébellion ou de préciosité et un acte de salut pour renforcer l'intrigue.

En lisant le livre à la lumière de la théorie ethnomidialogique, on remarque un plus grand potentiel d'assimilation et de connexion proposé par Yamã pour son travail à partir du récit des mythes. Nous observons ainsi les structures fonctionnelles de la littérature 
indigène qui contribuent à la popularisation du savoir indigène. Et nous pouvons certainement affirmer que la popularisation de ces connaissances, via la diffusion de livres, est une caractéristique de l'ethnomidialogie, qui, à son tour, cherche à comprendre comment les liens interpersonnels des peuples précolombiens peuvent être construits et établis par la contemporanéité.

Nous mettons ensuite en lumière une question concernant les animaux rivaux ayant un statut humain, en évaluant la rivalité de longue date entre les clans gavião et socó. Elle découle de la trahison de la femme de Tawatómiyt, le Gavião-Humain, qui était mariée à une Sokó'ria. Un jour, une voisine du clan socó est tombée amoureuse de la femme du Gavião, et à partir de ce moment, la voisine se cachait chaque jour de son mari Sokó'ria pour commettre l'acte de trahison. Lorsque Tawatómiyt partait à la chasse, le voisin revenait à la maison pour vivre comme l'amant de sa femme et, après tant de rencontres, celle-ci tombait enceinte et donnait naissance à un enfant qui n'aimait manger que du poisson, nourriture commune du clan socó.

Jusqu'à ce moment, le Gavião-Humain ne savait pas ce qui se passait dans sa maison, et quand il est sorti pour chasser, une araignée l'a accompagné dans sa chasse. L'araignée a dit que la femme de Tawatómiyt était tombée enceinte d'un autre garçon. Le Gavião-Humain l'a remercié et lui a offert une partie de sa chasse. Pour confirmer ce qu'il avait entendu, il a décidé de se cacher pour attraper la trahison et l'inattendu s'est produit. Le voisin Socó a apporté du poisson à sa maitresse et l'a posé sur ses genoux.

Gavião-Humain est descendu en furie et a déchiré son voisin en deux, a pris sa femme et l'a emmenée au domicile de Sukuyú'wéra, la mère des serpents, pour être son esclave à vie. Tawatómiyt a pris l'enfant et l'a prise en charge et nourri. Depuis évènement, il n'y a jamais eu de paix entre les clans des gaviões et celui des socós. Connaissant la mort de la femme Sokó'ría, ses frères se sont révoltés et ont décidé de tuer le Gavião-Humain. Soif de vengeance, ils ont planifié le meurtre au bord d'un igapó. Et c'est ce qu'ils ont fait. Après avoir noyé le Gavião, les frères ont fait la fête, mais le Tawatómiyt a laissé un fils et au fil des ans, le garçon a découvert la vérité sur la mort de son père et a décidé de se venger. Le Garçon-Gavião a grandi et créé beaucoup de force. Après que la tortue amie du garçon a invité ses oncles à planter du manioc, le Gavião a piqué et percé tout le monde avec ses griffes et les a tués. Ce jour-là, il y a eu un massacre parmi les Sokó'ria pour se venger de la mort de leur père Gavião-Humain, Tawatómiyt. 
Une fois de plus, nous remarquons que la stratégie de l'ethnomidialogie, qui consiste à construire une intrigue récurrente, avec un héros, un antagoniste ou un antihéros, un acte de rébellion ou de préciosité et un acte de salut ou de rédemption, est réalisée comme point central du récit. Et une fois de plus l'animisme, la relation entre l'animal et l'homme, est la condition du dialogue entre eux, exalté comme un facteur pour que le lecteur s'intéresse au contenu de l'œuvre. L'ethnomidialogie, donc, aide à la construction de supports pour gagner l'attention du public, indique principalement comment « capter » ce lecteur et le rendre intéressé par l'œuvre.

Le troisième chapitre raconte des fables de caractéristiques littéraires avec des compositions en vers ou en prose. Et encore une fois les personnages sont des animaux avec des aspects humains. Ils apportent des leçons de morale avec un contenu éducatif pour représenter la vie, avec un langage simple, que l'on trouve généralement dans la littérature pour enfants. Voici une autre perspective de l'écriture à partir de l'ethnomidialogie, qui établit un parallèle entre la question autochtone et la transmission d'informations à un public plus large.

L'histoire du tatou et du jaguar décrit qu'un jour les animaux sauvages ont accepté de voyager et que le jaguar, qui aimait se moquer de tous les animaux, a beaucoup attisé le tatou juste pour avoir le prétexte de le manger. Mais comme le tatou était intelligent, il ne donnait aucune confiance aux provocations et suivait son chemin. Lorsqu'il s'est approché d'une tanière, le tatou a eu l'idée de se venger du jaguar et a commencé de se moquer de lui. Pour essayer de l'enclencher, il a enfoncé sa tête dans la tanière et le tatou, qui s'était caché dans le trou, le tatou a donné un coup de pied dans le jaguar. Il est finalement devenu aveugle avec la force du coup. Inquiète, le jaguar ne savait plus comment vivre sans voir et a demandé l'aide d'autres animaux, mais aucun ne l'a aidé. Les animaux se sont moqués de lui à cause de la malveillance intense qu'il avait provoquée. Après des tentatives infructueuses, le jaguar n'a pas pu obtenir l'aide des animaux qui croisaient son chemin et a donc vécu un certain temps dans les bois.

Un jour, il a demandé l'aide de l'inambu, un oiseau tropical, qui a décidé d'aider le pauvre jaguar. Il était très maigre parce qu'il avait du mal à se nourrir. L'oiseau est allé chercher quelque chose et a gratté des écorces de jatobazeiro, faisant du médicament avec du jutaicyta. Puis il a rassemblé deux noyaux d'œil-de-akawã, au centre de la forêt. Avec le lait de jutaicyta, il a collé les yeux du jaguar avec les noyaux et avec le thé des écorces du 
jatobazeiro il a lavé les rétines de l'animal. Le jaguar reconnaissant a offert ses pintes et son cuir à l'inambu, et chaque fois que l'oiseau écoute les pas du jaguar, il chante pour ne pas être attaqué et est donc connu sous le nom d'horloge-inambu.

La médialogie permet de comprendre ce passage à travers deux prismes: la vengeance et la gratitude, qui sont des comportements antagonistes, mais équidistants, qui apparaissent dans la fable pour montrer aux lecteurs comment un homme ou un animal vraiment mauvais peut changer d'attitude et agir correctement. La fable, par elle-même édifiante, montre à quel point l'organisation narrative est orientée pour servir des compositions liées à l'organisation sociale ethnique des Sateré-Mawé. Et c'est précisément l'hypothèse de l'ethnomidialogie, qui s'intéresse à la vérification des processus de transmission de l'information des peuples traditionnels à la société urbaine.

\section{L’Implication Théorique des Résultats}

En analysant le livre en évidence, nous voyons qu'il s'est avéré nécessaire de corréler l'approche théorique de l'ethnomidialogie avec ce que soulignent les études communicationnelles impliquant la littérature contemporaine. Par nécessité, nous pourrions souligner Propp (2000), qui affirme à quel point les contes, romans, romans ou poèmes populaires s'inscrivent, d'une part, dans le courant folkloriste de l'anthropologie et dans l'approche littéraire des récits ethniques, comme nous essayons de le souligner dans le cadre de l'ethnomidialogie. Le travail de l'auteur (Id., op. cit.) sur la structure des contes fantastiques basés sur la vie quotidienne vécue réside dans le plan du contenu.

L'analyse de Propp est une recherche autonome de la forme d'expression des histoires contenues dans les chapitres rapportés, c'est-à-dire du point de vue de la trame ou de l'intrigue, celles-ci peuvent être considérées comme identiques. À partir de l'hypothèse, l'approche dominante pour inférer des propositions sur Sehaypóri.., c'est-à-dire l'ethnomidialogie, indique des conjoncturités littéraires. En ce qui concerne Propp (Id., op. cit.), la tâche de communication imbriquée dans le champ littéraire, par ce supposé, est de découvrir et de codifier les relations qui articulent les modèles observés, déjà ce qui classe les unités narratives minimales est l'action des personnages, l'action qui donne son nom à la fonction.

L'action des personnages, dans Sehaypóri... s'intègre directement dans l'intrigue narrative et est indépendante des personnes ou des animaux qui se trouvent dans les textes 
remplissant parfois la même fonction qui pouvait autrefois être exécutée par différents personnages. Les fonctions représentent des éléments essentiels des contes et fables du livre et forment l'action de la communication en mode narratif. De plus, il y a des éléments dans l'œuvre qui sont d'une grande importance, même s'ils ne déterminent pas le déroulement des trames. Et pourtant: les fonctions ne se succèdent pas toujours, car l'information est entrecoupée entre les activités les plus diverses.

Les principales qualités que doivent posséder les unités narratives sont: i) la constance, ii) l'invariance, iii) la limitation et iv) la comparabilité. Cependant, tous les contes et fables ne manifestent pas de telles fonctions dans leur intégralité. L'ordre dans lequel elles se produisent reste inchangé et deux modèles dominants d'articulation des fonctions manifestées au cours de l'intrigue peuvent être prédits: le combat contre l'agresseur et la victoire du héros et la tâche difficile et son accomplissement.

En association avec l'ethnomidialogie, nous avons pu voir que les fonctions des personnages sont des éléments constants et répétés du livre. Les rôles assignés aux personnages concrets, ainsi que leurs comportements, sont également toujours les mêmes. Chacun des personnages a le champ d'action, de sorte que toutes les fonctions ne sont pas obligatoires, mais en principe une fonction en entraine une autre.

Et des fonctions de personnages représentent des parties fondamentales des contes et des fables et doivent donc être isolées. Par fonction, on entend l'action d'un personnage défini du point de vue du sens au cours de l'intrigue. Propp (2000) déclare que:

[...] les éléments constants et permanents du conte sont les fonctions des personnages, quels que soient ces personnages et quelle que soit la manière dont ces fonctions sont remplies. Les fonctions sont les éléments constitutifs fondamentaux du conte ; le nombre de fonctions d'histoires courtes est limité ; la succession des fonctions est toujours identique, ces lois ne se réfèrent qu'au folklore; tous les contes merveilleux appartiennent au même genre en ce qui concerne la structure (PROPP, 2000, p. 59).

Les études structurales des aspects du livre étaient des conditions nécessaires à la recherche historique sur des textes de même ampleur. Concernant ce qui est décrit, Propp (2000) souligne que les personnages narratifs peuvent être regroupés en sept sphères. Ces sphères sont également mises en évidence au sein de l'ethnomidialogie, mais en tant que catégories de la socioculture impliquée. 
Pour Propp, la première est la sphère de l'agresseur, elle se produit quand il y a des méfaits, il y a des combats et d'autres formes de lutte contre le héros et sa poursuite. La deuxième est celle du donneur ou du pourvoyeur, qui comprend la préparation de la transmission de l'objet magique. La troisième sphère consiste dans l'action de l'auxiliaire qui comprend le déplacement du héros dans l'espace, la réparation des actes répréhensibles ou de l'erreur, l'aide pendant la persécution, la transfiguration du héros. Le quatrième est l'action de la princesse, le personnage recherché. La cinquième sphère d'action est celle de l'agent, qui se produit lorsque le héros est envoyé. La sixième sphère est l'action du héros qui se produit quand il va à la demande, la réaction aux exigences du donateur, le mariage. Et la dernière sphère est l'action du faux héros (Id., op. cit.).

Dans le contexte de l'ethnomidialogie, la socioculture établie dans la vie des SateréMawé peut être appréhendée en fonction du quotidien figuratif dans les écrits de Yaguarê. Il s'agit d'une situation de base littéraire et de contexte communicationnel, d'où il ressort que la communication et l'existence narrées ne sont pas des pôles équidistants. Et ce qui nous montre dans son récit ethnomidialogique, les sphères thématiques du livre sont contenues entre les caractérisations de personnages et se produisent suivant de telles possibilités: premièrement, la sphère d'action correspondante au personnage, deuxièmement, un personnage occupe plusieurs sphères d'action, et, enfin, dans le cas contraire, une sphère d'action est divisée entre plusieurs personnages. Il existe trois caractéristiques de personnages auxiliaires: i) universels, ii) assistants partiels et iii) assistants spécifiques. Chaque type a sa propre façon d'entrer dans la scène, ce qui correspond à des processus particuliers utilisés pour participer à des situations d'intrigue.

Ainsi, nous constatons que, dans l'ouvrage, Yaguarê a rapporté une connaissance de la nature amérindienne à travers une particularité, celle de l'universalisme. Cette caractéristique a été primordiale pour étudier le livre, car, selon le concept d'ethnomidialogie, la trajectoire du récit de l'auteur peut être particulière, mais les résultats recherchés par lui, qui en vérité fait une critique du « folklore » (dans le sens de diminuer coutumes et histoire) de savoirs autochtones, sont globaux, totalisants, applicables au contexte de l'indianisme amazonien contemporain. 


\section{Conclusion}

Connaissant l'importance de la diffusion des savoirs ethniques, constitués par la connaissance orale, par la communication sociale et les difficultés rencontrées dans la trajectoire des narratives des peuples autochtones, il a été possible de trouver dans l'ouvrage un langage simple mais très bien structuré. Sehaypóri..., de Yaguarê, présente des historicités ancestrales, des mythes sacrés, des légendes et des fables d'un peuple originaire d'Amérique précolombienne, avec une production contextuelle directement liée à des oralités présentes dans les textes.

La présentation de la mythologie du peuple Sateré est assez claire dans sa conception historique, et nous notons donc, dans le Sehaypóri.., qui signifie « recueil de mythes », ce qui a été souligné avant tout, ce sont des symboles et des territorialités remarquables des peuples autochtones, tel quel s'est exalté le Puratig. De cette rame sacrée nait toute une trajectoire historique des coutumes, savoirs et croyances du peuple SateréMawé. Cet exemple est juste pour avoir une idée de ce que nous remarquons dans la recherche: l'importance du regard indigène lui-même sur son histoire. Ce comportement, celui d'être l'auteur de sa propre histoire, reflète un changement radical de pensée et d'approche. Beaucoup de littérature amazonienne est (et a été pendant des années) écrite par des personnes étrangères à l'endroit, mais cela a changé.

L'approche native, né de l'oralité historique, est différenciée. À travers ses fables, mythes et paraboles, le but de cette nouvelle littérature narrée ne se limite pas à l'un ou l'autre groupe autochtone, mais présente des formes d'éducation pour différents peuples et différentes sociétés, créant des liens de respect pour la sagesse millénaire indigène, comme cela a été donné dans le cas du peuple Sateré-Mawé, qui brise les paradigmes et les préjugés. La socio-culture dépend de ce contraste avec le mythe, la légende et la cosmologie, qui servent de support à la diffusion et à la perpétuation des savoirs traditionnels d'un peuple.

Par conséquent, la littérature ethnique contribue à la croissance de l'interaction communicationnelle des peuples autochtones, de riche connaissance, avec d'autres populations du pays et du monde. Pour cela, il est nécessaire de stimuler l'interactivité avec différents espaces de l'archétype projeté dans la société. Au milieu d'une «ère de l'information multimédia », la présentation de ces sociétés inconnues au monde blanc en général permet des changements constants dans l'approche de la connaissance de chaque culture, dans des espaces différents et avec une charge gigantesque de savoirs traditionnels. 
Comprendre chaque individu selon sa propre conception des connaissances et des actes, c'est se connaitre soi-même et trouver des voies jamais parcourues par l'humanité, car les êtres humains font bouger le monde à travers des questions et en répondant aux problématiques posées pour créer un nouveau monde à leur époque. En ce qui concerne l'ethnomidialogie, nous pouvons comprendre que la littérature pratiquée par Yaguarê est un style d'échange d'informations dans lequel les lettres/littérature et la communication démocratique de l'information sont utilisées pour diffuser la connaissance ethnique et ce en soi (ce style) tend également à être un gain important dans la publicité des cosmologies indigènes, qui sont en résumé formatrice de l'ethos des ethnies.

\section{Références}

ALBUQUERQUE, R.; JUNQUEIRA, C. Brincando de onça e de cutia entre os Sateré-Mawé. Manaus: EDUA, 2017.

ABRIC, J. C. A zona muda das representações sociais. In: OLIVEIRA, D. C.; CAMPOS, P. H. F.(org.). Representações sociais, uma teoria sem fronteiras. Rio de Janeiro: Museu da República, 2005.p. 22-34.

BARDIN, L. Análise de conteúdo. Lisboa: Edições 70, 2002.

FERREIRA, R. A. A etnomidialogia e a interface com o politicamente correto. Extraprensa, São Paulo, ano IV, n. 10, p. 1-18, jul. 2012.

FERREIRA, R. A. A formação do jornalista na abordagem dos fenômenos da diversidade e dos grupos minorizados: uma perspectiva didático-pedagógica da educomunicação e etnomidialogia. Extraprensa, São Paulo, ano 2010, n. 3, p. 321-344, 2015. Edição especial.

IANNI, O. Sociedade global, história e transculturação. In: TAVARES DOS SANTOS, J. V. (org.). Violências em tempo de globalização. São Paulo: Hucitec, 1999. p. 43-65.

MENDONÇA, M. S.; FRANÇA, J. F.; OLIVEIRA, A. B.; PRATA, R. R.; AÑEZ, R. B. S. Etnobotânica e o saber tradicional. In: FRAXE, T. J. P.; PEREIRA, H. S.; WITKOSKI, A. C. (org.). Comunidades ribeirinhas amazônicas: modos de vida e uso dos recursos naturais. Manaus: EDUA, 2007, p. 91-103.

PROPP, W. A morfologia do conto maravilhoso. 4. ed. Lisboa: Vega Universidade, 2000.

SPERBER, D.; WILSON, D. Relevance: communication and cognition. Cambridge: Blackwell,1995.

VALA, J. Análise de conteúdo. In: SILVA, A.; PINTO, J. (org.). Metodologia em Ciências

Sociais. Porto, Afrontamento, 1986. p. 101-128. 
VIVEIROS DE CASTRO, E. Cosmological perspectivism in Amazonia and elsewhere: four lectures given in Department of Social Anthropology. Chicago: HAU, 1998.

VIVEIROS DE CASTRO, E. Filiação intensiva e aliança demoníaca. Novos Estudos, São Paulo, n. 77, p. 91-126, 2007.

VOSOUGHI, S.; ROY, D.; ARAL, S. The spread of true and false news online. Science, Washington, v. 359, p. 1146-1151, 2018.

WAGNER, R. The invention of culture. Chicago: The University of Chicago Press, 2010.

\title{
From orality to narrative: the case of the sacred book of the indigenous Sateré-Mawé of the Amazon
}

\begin{abstract}
The aim was to (i) characterize the narrative and communication process, born of orality, a book reference of the Sateré-Mawé and (ii) perform interpretative readings and analyzes of the texts, with content analysis, considering the oral communication and speech. These two points are described in the "Theoretical Approach" sections and in the "Results and Discussion" section. The presentation of the mythology of the Sateré-Mawé is clear in its historical conception, because in the Sehaypóri..., which means "collection of myths", what we underlined was main symbols and the way in which these people build their memories other times transmitted orally.
\end{abstract}

\section{Keywords}

Orality; Narrative; Literature;Sateré-Mawé/AM; Amazon

\section{Da oralidade à narrativa: o caso do livro sagrado dos indígenas Sateré-Mawé da Amazônica}

\section{Resumo}

O objetivo foi (i) caracterizar o processo narrativo e comunicacional, nascido da oralidade, de uma obra sagrada, de referência do Sateré-Mawé, e (ii) realizar leituras interpretativas e análises dos textos do livro, com análise de conteúdo, considerando a comunicação oral e o discurso. Esses dois pontos estão descritos nos títulos "Abordagem Teórica" e na seção 
"Resultados e Discussão". A apresentação da mitologia dos Sateré-Mawé é clara na sua concepção histórica, pois no Sehaypóri..., que significa "coleção de mitos", o que se enfatizou foram os símbolos e a forma como este povo construiu as suas memórias no passado.

\section{Palavras-chaves}

Oralidade; Narrativa; Literatura; Sateré-Mawé/AM; Amazônia

\section{Autoria para correspondência}

Renan Albuquerque

renanalbuquerque@ufam.edu.br

\section{Como citar}

ALBUQUERQUE, Renan; VASCONCELOS, Fabrício; BUSARELLO, Flávia Roberta. De l'oralité au récit: le cas du livre sacré des indigènes Sateré-Mawé, en Amazonie. Intexto, Porto Alegre, e109699, jan./dez. 2021. DOI: http://dx.doi.org/10.19132/1807-8583202152.109699

Recebido em 08/12/2020

Aceito em 07/07/2021 